\title{
Opisthorchiasis, Acetaminophen Overdose and Cholangiocarcinoma : Correspondence
}

Asian Pac J Cancer Prev, 23 (1), 1-1

\section{Dear Editor}

we read the article entitle "Acetaminophen Overdose Enhances Early Cholangiocarcinoma (CCA) in Opisthorchiasis Hamsters" with a great interest (Sriraj et al., 2021). Sriraj et al. concluded that "combined intake of liver flukecontaminated raw fishes and acetaminophen rendered more severity of CCA than sole consumption of the contaminated raw fishes (Sriraj et al., 2021)." CCA is an important cancer seen in many developing countries. The high incidence of this cancer is seen in Southeast Asia and it is proposed for relationship with opisthorchiasis. Regarding liver problems, several infectious pathogens, drugs and toxic substances can induce liver abnormality. In the present report, acetaminophen overdose and parasite are studied as hepatotoxic factors and it is not surprising that there is a more liver problem in the group with more number of primary hepatotoxic factors than the other group with less number or no hepatotoxic factor. The conclusion in the present study might be extrapolated. It should conclude on only hepatitis problem following receiving different numbers of hepatotoxic factors but it should not conclude on the severity of CCA since no animals in this experiment had no CCA. Regarding acetaminophen over dose, it is not an uncommon problem in clinical practice and the problem might exist in any developing countries that local people are not well educated and there is no good control of drug prescription. Regardless of cancer, acetaminophen overdose can cause clinical problem to liver. For cholangiocarcinoma patients, the chance of liver metabolism might occur and excretion of acetaminophen might be altered (Alsaleh et al., 2019). A good control of drug dosage for the patient with CCA is important. Finally, regarding role of acetaminophen for caring patients with CCA, if it is used in a good dosage control, it might still be useful. At least, it can be a good and proven safe drug for palliative care of patients after therapeutic procedures (Carrafiello et al.,2010).

Conflict of interest None

\section{References}

Alsaleh M, Sithithaworn P, Khuntikeo N, et al (2019). Characterisation of the Urinary Metabolic Profile of Liver Fluke-Associated Cholangiocarcinoma. J Clin Exp Hepatol, 9, 657-75.
Carrafiello G, Laganà D, Cotta E, (2010). Radiofrequency ablation of intrahepatic cholangiocarcinoma: preliminary experience. Cardiovasc Intervent Radiol, 33, 835-9

Sriraj P, Boonmars T, Aukkanimart R, et al (2021). Acetaminophen Overdose Enhances Early Cholangiocarcinoma in Opisthorchiasis Hamsters. Asian Pac J Cancer Prev, 22, 3903-12.

\section{Rujittika Mungmunpuntipantip ${ }^{1 *}$, Viroj Wiwanitkit ${ }^{2,3}$}

${ }^{1}$ Private Academic Consultant, Bangkok Thailand. ${ }^{2}$ Honorary Professor, Dr DY Patil University, Pune, India; visiting professor, Hainan Medical University, Haikou, China. ${ }^{3}$ Distinguished Professor, Parasitic Disease Research Center, Suranaree University of Technology, Nakhon Ratchasima, Thailand. *For Correspondence: rujittika@gmail.com 